\title{
Reviewer acknowledgement 2013
}

Satoshi Gando

\section{Contributing reviewers}

The editors of Journal of Intensive Care would like to thank all our reviewers who have contributed to the journal in Volume 1 (2013).

\section{Toshikazu Abe Japan}

Fumimasa Amaya

Kohkichi Andoh

Japan

Daniele Bollero

Italy

Moritoki Egi

Japan

Yuji Fujino

Japan

Seitaro Fujishima

Japan

Satoshi Fujita

Japan

Shigeki Fujitani

Japan

Satoru Hanada

Japan

Satoru Hashimoto

Japan

Mineji Hayakawa

Japan

Yoshiro Hayashi

Australia

Hirofumi Hino

Japan

\author{
Toshiaki Ikeda \\ Japan
}

Hideaki Imanaka

Japan

Hideo Inaba

Japan

Hironori Ishihara

Japan

Eiji Isotani

Japan

Takashi Ito

Japan

Gavin Joynt

Hong Kong

Motoshi Kainuma

Yasuyuki Kakihana

Japan

Masato Kato

Japan

Nobuyuki Katori

Japan

Atsushi Kawaguchi

Canada

Kaneyuki Kawamae

Japan

Tatsuya Kawasaki

Japan
Kosaku Kinoshita
Japan

Yutaka Kondo

Japan

Yoshifumi Kotake

Japan

Joji Kotani

Japan

Toru Kotani

Japan

Yasuhiro Kuroda

Japan

Jeffrey Lipman

Australia

John Marshall

Canada

Naoyuki Matsuda

Japan

Hikoro Matsui

Japan

Chieko Mitaka

Japan

Hiroshi Morimatsu

Japan

Yuji Morimoto

Japan

Hiroshi Morisaki

Japan

Correspondence: gando@med.hokudai.ac.jp

Division of Acute and Critical Care Medicine, Department of Anesthesiology

and Critical Care Medicine, Hokkaido University Graduate School of Medicine,

Hokkaido, Japan 
Kiyoshi Moriyama

Japan

Takaya Morooka

Japan

Satoshi Nakagawa

Japan

Susumu Nakagawa

Japan

Masaki Nakane

Japan

Minoru Nakano

Japan

Koichi Nakao

Japan

Hiromichi Narumiya

Japan

Shinichi Nishi

Japan

Hiroshi Nishida

Japan

Masaji Nishimura

Japan

Takeshi Nomura

Japan

Hiroshi Nonogi

Japan

Shin Nunomiya

Japan
Shigeto Oda

Japan

Kazufumi Okamoto

Japan

Mutsuo Onodera

Japan

Kiyohiro Oshima

Japan

Luc Puis

Belgium

Yoshiro Sakaguchi

Japan

Masamitsu Sanui

Japan

Nobuo Sasaki

Japan

Nobuaki Shime

Japan

Yoshito Shiraishi

Japan

Kazuya Sobue

Japan

Naosuke Sugai

Japan

Takeshi Suzuki

Japan

Yoshio Tahara

Japan
Shinhiro Takeda

Japan

Tetsuhiro Takei

Japan

Naoshi Takeyama

Japan

Ryosuke Tsurura

Japan

Osamu Umegaki

Japan

Yutaka Usuda

Japan

Masahiko Uzura

Japan

Hiroyuki Watanabe

Japan

Hiroshi Yamada

Japan

Takeshi Yamamoto

Japan

Hidekazu Yukioka

Japan

doi:10.1186/2052-0492-2-4

Cite this article as: Gando: Reviewer

acknowledgement 2013. Journal of Intensive

care 2014 2:4. 